\title{
Meibomian gland imaging: A review
}

\begin{tabular}{|c|}
\hline $\begin{array}{l}\text { Authors: } \\
\text { Deanne L. Nicholas } \\
\text { Wayne D.H. Gillan }\end{array}$ \\
\hline $\begin{array}{l}\text { Affiliations: } \\
{ }^{1} \text { Department of Optometry, } \\
\text { University of Johannesburg, } \\
\text { South Africa }\end{array}$ \\
\hline $\begin{array}{l}\text { Correspondence to: } \\
\text { Deanne Nicholas }\end{array}$ \\
\hline $\begin{array}{l}\text { Email: } \\
\text { deannenic@gmail.com }\end{array}$ \\
\hline $\begin{array}{l}\text { Postal address: } \\
\text { PO Box 17011, Doornfontein } \\
\text { 2028, South Africa }\end{array}$ \\
\hline $\begin{array}{l}\text { Dates: } \\
\text { Received: } 03 \text { Feb. } 2014 \\
\text { Accepted: } 17 \text { Nov. } 2014 \\
\text { Published: } 30 \text { Mar. } 2015\end{array}$ \\
\hline $\begin{array}{l}\text { How to cite this article: } \\
\text { Nicholas DL, Gillan, WDH. } \\
\text { Meibomian gland imaging: A } \\
\text { review. Afr. Vision Eye Health. } \\
\text { 2015;74(1); Art. \#12, } 4 \text { pages. } \\
\text { http://dx.doi.org/10.4102/ } \\
\text { aveh.v74i1.12 }\end{array}$ \\
\hline $\begin{array}{l}\text { Note: } \\
\text { This article results from } \\
\text { preliminary research } \\
\text { towards a Masters degree } \\
\text { in optometry undertaken } \\
\text { by D.L. Nicholas under the } \\
\text { supervision of Prof. W.D.H. } \\
\text { Gillan. }\end{array}$ \\
\hline $\begin{array}{l}\text { Copyright: } \\
\text { (C) 2015. The Author(s). } \\
\text { Licensee: AOSIS } \\
\text { OpenJournals. This work is } \\
\text { licensed under the Creative } \\
\text { Commons Attribution } \\
\text { License. }\end{array}$ \\
\hline
\end{tabular}

\section{Read online:}

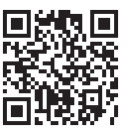

Scan this QR code with your smart phone or mobile device to read online.
The meibomian glands of the upper and lower eyelids play a valuable role in secreting the lipid layer of the tear film. Disturbances in meibomian gland function may result in altered secretion and variations in tear composition which may lead to meibomian gland dysfunction and evaporative dry eye, leading to ocular discomfort. To diagnose and monitor the structural and functional changes occurring within the glands of the eyelids, various imaging techniques are available. Some of the methods used to evaluate the tears and therefore the meibum within the tears include evaporimetry, interferometry, tear osmolarity and meibometry. With these techniques, changes in the lipid layer of the tear film can be quantified and alterations in meibomian gland function assessed. Meibography is an additional method that can be used; it has the unique feature of allowing the assessment of meibomian gland morphology during ocular surface disease processes. The aim of this review is to create an improved understanding of the meibomian glands and the ways that they may be investigated in order to expand on the treatment methods available.

\section{Introduction}

Meibomian glands may be classified as holocrine glands embedded within the tarsal plate of both the superior and inferior lid margins. ${ }^{1}$ Individual meibomian glands consist of a central duct that passes through the length of the gland. ${ }^{2}$ Extending from this central duct are glandular structures termed acini. ${ }^{1,2}$ The acini are minute grape-like structures which contain modified sebaceous cells known as meibocytes. ${ }^{3}$ Meibocytes are responsible for the synthesis of a lipid secretion known as meibum, ${ }^{4}$ which passes through numerous ductules into the central canal. ${ }^{2}$ From the central canal, the meibum is expelled onto the eyelid margin via a compressive force, and forms the superficial layer of the tear film. ${ }^{1,2}$

The layer of meibum within tears forms an integral part of the tear film and has various important functions. The meibum secretion is responsible for stabilising the tear film and providing a hydrophobic barrier, thereby preventing excessive evaporation of tears from the ocular surface. ${ }^{1,2,5}$ Meibum also provides lubrication, thus preventing ocular irritation and in turn affording a smooth optical surface. ${ }^{1,25}$ Antimicrobial properties are also present within the meibomian secretion, providing protection against external antigens that may come into contact with the ocular surface. ${ }^{1}$

Meibomian gland function is thought to be regulated by neuronal, hormonal and vascular factors. ${ }^{1}$ Abnormalities within these regulating mechanisms, or disturbances in the functioning of the meibomian glands, may lead to meibomian gland disease which can take on various forms. ${ }^{6}$ There may be an absence or complete deficiency of the meibomian glands that may occur as a congenital condition. ${ }^{6}$ In some cases, the meibomian glands may be replaced by an extra row of eyelashes, a condition known as dystichiasis ${ }^{6}$ which may be inherited or acquired. The remaining glands may be enlarged or distorted. Lastly, the most common form of meibomian gland disease is known as meibomian gland dysfunction (MGD), where changes may occur in the ducts, orifices, acini and secretory activities of the glands. ${ }^{6}$

The various structural changes which take place during meibomian gland disease can be assessed using numerous imaging techniques. One of these imaging methods, termed meibography, is a technique that allows assessment of the morphology of individual meibomian glands. ${ }^{3}$ Using the images obtained, a qualitative analysis can be done based on the structural changes taking place, permitting the diagnosis of dry eye disease. ${ }^{3}$ Some of these structural changes include loss of glandular tissue (gland dropout), dilation of the ducts within the glands, and stasis of the meibum. ${ }^{7}$

\section{Meibomian gland dysfunction}

MGD or posterior blepharitis can be defined as an abnormality occurring in the functions of the meibomian glands owing to either obstruction or changes in the secretory activities of the glands. 3,8 MGD can be classified as primary or secondary to systemic diseases; it can be focal or diffuse and 
may lead to symptoms of ocular irritation, alteration of the tear film or inflammation of the meibomian gland orifices. ${ }^{3}$ Numerous structural changes can take place in MGD that will result in the functions of the glands being altered. ${ }^{6}$

Some of the structural changes occurring within the lids may include thickening, rounding or distortion of the lid margin owing to obliteration of the meibomian gland orifices. ${ }^{6}$ The mucocutaneous junction of the lid may become irregular, occasionally it may be displaced posteriorly or it may become elevated. ${ }^{6}$ The orifices of the meibomian glands may change in number owing to the disease process, they may duplicate or reduce in number, and become pouted or narrowed. ${ }^{6}$ The meibomian glands may be expressed (by applying digital pressure to the lids) and the secretions be assessed; the secretions may be classified as clear, cloudy, granular or inspissated (toothpaste consistency). ${ }^{6}$ These changes can be seen in meibomian gland disease where the meibomian glands do not function efficiently or where a disturbance exists within the functional mechanisms of these glands.

Obstructive MGD is the most common form of MGD and may result in hyposecretion or the absence of lipid-rich meibum, which may lead to excessive and rapid evaporation of the tear film. ${ }^{3,9}$ Increased evaporation of the tear film may result in the inability to tolerate contact lens wear, an unstable tear film or blepharitis. ${ }^{10}$ Other forms of meibomian gland dysfunction include meibomitis, meibomian neoplasia and meibomian seborrhoea. ${ }^{6}$ The seborrheic type of MGD results from hypersecretion of meibum, which induces an inflammatory reaction resulting in ocular irritation. ${ }^{3}$ It has not yet been established whether the seborrheic form results from true hypersecretion of meibum or the accumulation of static meibum that is expressed from the ductules. ${ }^{6,8}$ The seborrheic form of MGD demonstrates normal tear evaporation rates as opposed to the obstructive form of MGD. ${ }^{8}$ Meibomitis can be defined as an inflammation of the meibomian gland orifices along with solidification of the meibum within the glands. ${ }^{6}$

The prevalence of MGD has been found to be approximately $39 \%-50 \%{ }^{8,11}$ in the general population worldwide and is one of the primary causes of evaporative dry eye. ${ }^{3,8}$ Dermatological diseases such as acne rosacea and psoriasis may be found in association with MGD. ${ }^{6,8}$ Owing to the prevalence of meibomian gland dysfunction, it has become increasingly important to accurately examine and assess the meibomian glands of dry-eye patients. There are various techniques available for assessment of the tears and the meibum it contains, namely evaporimetry, interferometry, tear osmolarity and meibometry. 3,8

\section{Meibography}

Meibography is a specialised imaging technique used to visualise the morphology of the meibomian glands. ${ }^{2,3,2,13}$ Wise et al. ${ }^{3}$ stated that this technique utilises ultraviolet light to transilluminate the glands of an everted eyelid. ${ }^{3}$ The meibomian gland structure can be viewed by placing the tip of a transilluminating light probe against the everted eyelid which is thus illuminated from behind. ${ }^{2}$ Both red and white light sources can be used to image the glands which can then be viewed through a slitlamp biomicroscope from which assessments can be made. ${ }^{7}$ The images are captured by an infrared photography system..$^{2,3}$ Meibography provides an in vivo method of assessing the morphology of the glands, allowing clinicians to observe any loss of glandular tissue as well as the meibum within the glands. ${ }^{7}$ This imaging method, which has been termed contact meibography, ${ }^{3}$ was considered very useful in the clinical environment; however, it was found to be time consuming and expensive, and caused patient discomfort owing to its invasive nature..$^{2,3}$ In 2008, non-contact meibography was introduced, a method in which an infrared light source is used, which does not come into contact with the eyelid of the patient and therefore results in less discomfort. ${ }^{3,7,14}$ An infrared charge-coupled device is attached to a slitlamp biomicroscope without the need for a probe. ${ }^{7}$ Conventional meibography allows only the observation of a small central region of the lid margin whereas non-contact meibography encompasses a larger, wider region of the upper and lower lids for extensive examination. ${ }^{3,5}$ The light and dark contrast is opposite to that of contact meibography, in that the glands appear light instead of dark when using the non-contact method. ${ }^{7}$ The upper and lower lids can be assessed to determine the degree
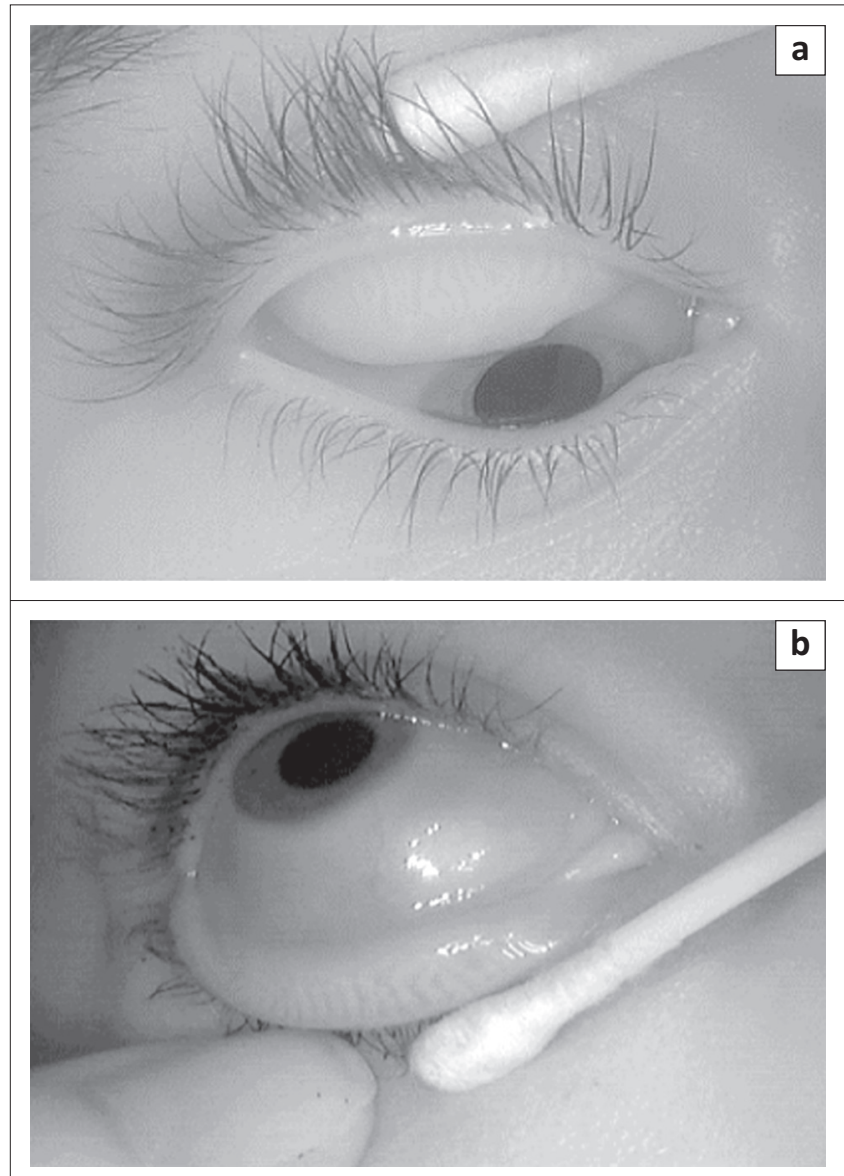

Source: Photos taken by W.D.H. Gillan

FIGURE 1: (a) The meibomian glands of an everted upper eyelid; distortion or non-linearity can be seen. (b) The appearance of normal meibomian glands seen within the lower lid margin. 
of meibomian gland dropout, which refers to the loss or destruction of glandular tissue. ${ }^{2,3}$ As demonstrated in Figure $1 \mathrm{a}$, the destruction of glandular tissue can be seen by the distortion or non-linearity within the glands. Normal meibomian gland structure can be seen in Figure 1b, where the glands have a regular and linear appearance.

In 2012, a mobile meibography system in the shape of a pen was introduced; the system uses an infrared LED attached to a handheld camera system. ${ }^{3}$ This is a convenient and simple method of assessing the glands as it is portable and eliminates the need for a slitlamp biomicroscope. ${ }^{3}$

The Oculus Keratograph 4 has become an important tool that can be used for contact lens assessment, corneal topography, measuring tear meniscus height, pupillometry as well as many other procedures. ${ }^{15}$ The Keratograph 4 may also be used to image the meibomian glands of the upper and lower lids. ${ }^{15}$ Using this multi-functional apparatus, both still and video images of the meibomian glands can be captured by means of an infrared illumination system that can be manually adjusted. ${ }^{2,15}$ These meibography methods enable an examiner to accurately assess the structural and functional changes that occur within the meibomian glands as these changes could presage MGD. To determine the extent of damage to the glands, the images obtained during meibography are compared with a series of gradings, ranging from mild to severe loss or damage. These grading scales enable the examiner to qualitatively assess the changes occurring during the disease process.

\section{Grading scales}

The extent of meibomian gland dropout has been shown to be directly correlated with ocular surface conditions., ${ }^{5,14}$ There are various factors to be considered when assessing the meibomian glands. These include the percentage of partial glands, the presence of chalazia and the number of glands present within the lid margin. ${ }^{7}$ In the presence of an ocular surface disease, the glands become enlarged and tortuous whilst the ducts appear to be dilated, eventually leading to complete destruction of the gland. ${ }^{3}$ The changes occurring within the structures of the meibomian glands can be qualitatively assessed using a grading scale., ${ }^{2,3}$ Three main grading methods have been identified: the meiboscore method, the meiboscale and the meibograde method.,12

The meiboscore method assesses the areas of partially or completely destroyed meibomian gland structure compared with the normal regions of the eyelid margin. ${ }^{3}$ The meibograph images are assessed and assigned a value proportional to the area of destruction, ranging from 0 to 3 , with 3 indicating that the involved area encompasses more than $66 \%$ of the lid margin. ${ }^{3}$ A score of 2 implies that the area of the lid margin involved encompasses between 33\% and $66 \%$. Less than $33 \%$ of the area is involved if a score of 1 is obtained, and 0 implies that the lid has no partial or missing glands. ${ }^{3}$ The numerical values for the upper and lower lids are summed together ${ }^{3}$ to obtain a score ranging from 0 to 6 .
The meiboscale is a five-grade pictorial scale used to grade meibography images, as opposed to previous scales where four different grades were used. ${ }^{12}$ This subjective method, developed by Pult, ${ }^{12}$ measures the area of meibomian gland $\operatorname{loss}^{12}$ from degree 0 to degree 4 . Degree 0 implies that there is no meibomian gland loss; degree 1 is indicative of $25 \%$ or less. Degree 2 indicates $26 \%-50 \%, 51 \%-75 \%$ is indicated by degree 3 , and degree 4 indicates loss of over $75 \%$ of the meibomian glands within the lid. ${ }^{12}$

The meibograde method provides a more comprehensive grading scale. ${ }^{3}$ It is based on three important structural changes that can occur within the meibomian glands: gland dropout, gland shortening and gland distortion. ${ }^{3,16}$ Each of these three categories is assigned a meiboscore ranging from 0 to 3 as mentioned above; thereafter the scores from the three individual categories are summed to obtain a total score ranging from 0 to 9 for each eyelid. ${ }^{3}$ These grading scales are extensively used and can be useful in the evaluation of MGD and evaporative dry eye. ${ }^{12}$

Studies have shown that meibomian gland dropout is found to be increased in patients wearing contact lenses and in patients of greater age., ${ }^{2,5}$ Dropout is also more commonly observed in glands of the lower lid as opposed to glands of the upper lid, ${ }^{2,5}$ which may be related to the different structural features of the upper and lower tarsus. ${ }^{5}$ The upper tarsus has been shown to be anatomically larger than the lower tarsus. ${ }^{5}$

\section{Other methods of imaging}

Laser confocal microscopy is a relatively new technology that can be used as a diagnostic tool in evaluating various factors involved in ocular surface diseases. ${ }^{2,3,17}$ One of the disadvantages of this imaging technique is that it invasive and the use of topical anaesthetic is necessary. ${ }^{17}$ Once the patient has been anaesthetised, the eyelid is everted and a Tomo-Cap is used to applanate the palpebral conjunctiva, thereby obtaining digital images of the meibomian gland structures. ${ }^{2,17}$ This method has the advantage of being able to resolve microscopic structures within the eyelid margin. ${ }^{17}$ Using this innovative technology, Matsumoto et al. ${ }^{17}$ performed a study in which two new parameters could be described relating to the meibomian glands, namely glandular acinar unit diameter and acinar unit density.,17 These parameters can now be studied extensively and quantitative measures obtained. Another parameter that can be obtained and is very useful in providing information regarding the severity of MGD is the periglandular inflammatory cell density $^{2}$ which allows the clinician to determine the extent of inflammation occurring within the glands during ocular surface disease processes.

Optical coherence tomography (OCT) is an ultrastructural imaging technique used in ophthalmic diagnosis. ${ }^{18}$ OCT can be used to obtain three-dimensional in vivo images of the meibomian glands; $;^{2,3}$ it is thus possible to produce an image from reflection of light off the desired surface being viewed, allowing formation of an image of the structure 
being studied. ${ }^{18}$ OCT has an important advantage over any of the other imaging techniques, in that it is able to quantify the structure of the meibomian glands volumetrically. ${ }^{3}$ These volumetric measures can be used to diagnose and follow the progression of MGD. ${ }^{3}$

\section{Conclusion}

The production of meibum, which is responsible for forming the superficial layer of the tear film, is a complex process that can be disturbed by alterations in the structure of the meibomian glands. These changes may result in meibomian gland dysfunction which is a primary cause of evaporative dry eye. The changes occurring in the tear film can be monitored using the various imaging techniques mentioned above. Meibography has been found to be the most comprehensive procedure for imaging the morphological features of the meibomian glands. Quantitative measures can be made and the progress of ocular surface diseases can be monitored. Meibography is not generally used in routine clinical examinations; however, owing to its specificity and sensitivity in the diagnosis of MGD, it is a useful tool that could be utilised more frequently.

\section{Acknowledgements Competing interests}

The authors declare that they have no financial or personal relationships which may have inappropriately influenced them in writing this article.

\section{Authors' contributions}

This article results from preliminary research towards a Master's degree in optometry undertaken by D.L.N. (University of Johannesburg), under the supervision of Professor W.D.H.G. (University of Johannesburg).

\section{References}

1. McCulley JP, Shine WE. Meibomian gland function and the tear lipid layer. Oc Surf. 2003;1:97-106. http://dx.doi.org/10.1016/S1542-0124(12)70138-6

2. Ngo W, Srinivasan $S$, Jones L. Historical overview of imaging the meibomian glands. J Optom. 2013;1:1-8. http://dx.doi.org/10.1016/j.optom.2012.10.001

3. Wise RJ, Sobel RK, Allen RC. Meibography: A review of techniques and technologies. Saudi J Ophthalmol. 2012;26:349-356. http://dx.doi.org/10.1016/j. sjopt.2012. 08.007

4. Butovich IA. The meibomian puzzle: Combining pieces together. Progr Reti Eye Res. 2009;28:483-498. http://dx.doi.org/10.1016/j.preteyeres.2009.07.002

5. Ban Y, Shimazaki-Den S, Tsubota K, Shimazaki J. Morphological evaluation of meibomian glands using noncontact infrared meibography. Oc Surf. 2013;11:4753. http://dx.doi.org/10.1016/j.jtos.2012.09.005

6. Bron AJ, Benjamin L, Snibson GR. Meibomian gland disease: Classification and grading of lid changes. Eye. 1991;5:395-411. http://dx.doi.org/10.1038/ eye.1991.65

7. Pult H, Nichols JJ. A review of meibography. Optom Vis Sci. 2012;89:760-769. http://dx.doi.org/10.1097/OPX.0b013e3182512ac1

8. Foulks GN, Bron AJ. Meibomian gland dysfunction: A clinical scheme for description, diagnosis, classification and grading. Oc Surf. 2003;1:107-126. $\mathrm{http}: / / \mathrm{dx}$.doi.org/10.1016/S1542-0124(12)70139-8

9. Yokoi N, Mossa F, Tiffany JM, Bron AJ. Assessment of meibomian gland function in dry eye using meibometry. Arch Ophthalmol. 1999;117:723-729. http://dx.doi. org/10.1001/archopht.117.6.723

10. Arita R, Suehiro J, Haraguchi T, Shirakawa R, Tokoro H, Amano S. Objective image analysis of the meibomian gland area. Brit J Ophthalmol. 2014;98(6):746-755. http://dx.doi.org/10.1136/bjophthalmol-2012-303014

11. Nien CJ, Massei S, Lin G, et al. Effects of age and dysfunction on human meibomian glands. Arch Ophthalmol. 2011;129:462-469. http://dx.doi.org/10.1001/archo phthalmol.2011.69

12. Pult $\mathrm{H}$, Riede-Pult B. Comparison of subjective and objective assessment in meibography. Contact Lens Ant Eye. 2013;36:22-27. http://dx.doi.org/10.1016/j. clae.2012.10.074

13. Alsuhaibani AH, Carter KD, Abramhoff MD, Nerad JA. Utility of meibography in the evaluation of meibomian glands morphology in normal and diseased eyelids. Saudi J Ophthalmol. 2011;25:61-66. http://dx.doi.org/10.1016/j.sjopt.2010.10.005

14. Pult $\mathrm{H}$, Riede-Pult BH. Non-contact meibography in diagnosis and treatment of non-obvious meibomian gland dysfunction. J Optom. 2012;5:2-5. http://dx.doi. org/10.1016/j.optom.2012.02.003

15. Srinivasan S, Menzies KL, Sorbara L, Jones LW. Imaging meibomian glands on a patient with chalazia in the upper and lower lids: A case report. Contact Lens Ant Eye. 2013;36:199-203. http://dx.doi.org/10.1016/j.clae.2013.02.014

16. Jester JV, Nicolaides N, Smith RE. Meibomian gland studies: histologic and ultrastructural investigations. Inv Ophthalmol Vis Sci. 1981;20:537-547.

17. Matsumoto Y, Sato EA, Ibrahim OMA, Dogru M, Tsubota K. The application of in vivo laser confocal microscopy to the diagnosis and evaluation of meibomian gland dysfunction. Mol Vis. 2008;14:1263-1271.

18. Ehlers JP, Gupta PK, Farsiu S, et al. Evaluation of contrast agents for enhanced visualization in optical coherence tomography. Inv Ophthalmol Vis Sci. 2010;51:6614-6619. http://dx.doi.org/10.1167/iovs.10-6195 